\title{
OPTICAL STUDIES OF THERMALLY ACTIVATED VERTICAL HOLE TRANSPORT IN ZnCdSe/ZnSSe SUPERLATTICE
}

\author{
A. Lebedev, S. Sorokin, A. Toropov, T. Shubina, N. Il'inskaya, \\ O. NeKrutkina, S. IVANov \\ A.F. Ioffe Physico-Technical Institute, 194021 St. Petersburg, Russia \\ G. Pozina, P. Bergman and B. Monemar \\ Linköping University, 58183 Linköping, Sweden
}

Miniband transport in alternatively-strained $\mathrm{ZnCdSe} / \mathrm{ZnSSe}$ short period superlattices is investigated using a structure with an enlarged quantum well. Temperature dependences of time-resolved and continuous wave photoluminescence have been measured, demonstrating an efficient temperature-induced vertical hole transport. A quantitative description is given for the carrier kinetics in these structures.

PACS numbers: 78.66.Hf, 78.45.+h, 71.35.-y, 78.47.+p, 72.80.Ey

\section{Introduction}

It has been recently suggested and experimentally shown that a short-period superlattice (SL) used in the active region of $\mathrm{ZnCdSe} / \mathrm{ZnSSe} / \mathrm{ZnMgSSe}$ separate confinement heterostructure lasers instead of the bulk waveguide layers can significantly improve the electronic confinement $[1,2]$. The SL waveguide lattice-matched to a GaAs substrate as a whole can easily be designed, practically for any $S$ and Cd content used in the $\mathrm{ZnMgSSe}$ cladding and $\mathrm{ZnCdSe}$ quantum well (QW) layers, and grown in a non-interrupted process without any variation of growth parameters [3]. The recent studies demonstrate that a variation of CdSe mole fraction from 0 to 0.27 allows one to cover the range of lasing wavelength from 523 to $470 \mathrm{~nm}$ with the room temperature threshold power densitics varied from $18 \mathrm{~kW} / \mathrm{cm}^{2}$ to $55 \mathrm{~kW} / \mathrm{cm}^{2}$, respectively [4]. To extend this approach to injection lasers, further research is needed, aimed at optimization of carrier tunneling transport along the growth direction of the SL, serving simultaneously as a carrier emitter.

Vertical transport of carriers in the short-period SLs is preferably performed along minibands with band width varied in a wide range by changing composition and thickness of the constituent layers. This phenomenon has been experimentally 
investigated for a long time by different research groups. Particularly, Chomette et al. have suggested to use an enlarged QW incorporated in the SL to study carrier localization in random fluctuations of the well width [5]. Utilizing the idea of an enlarged QW for a study of carrier transport, Nakamura et al. have developed a simple rate equations model which effectively describes the kinetics of photoexcited carriers in a GaAs/AlAs system $[6,7]$.

In this paper, we present optical studies of transport properties of alternatively-strained $\mathrm{ZnSSe} / \mathrm{ZnCdSe}$ short-period SLs. The ZnSe-based structures have a $n$-type residual background doping $\left(N_{\mathrm{D}} \approx 10^{15} \mathrm{~cm}^{-3}\right)$, therefore the holes created by laser excitation determine the recombination processes in the structures. The efficiency of the vertical hole transport has been studied by means of PL experiments both in continuous wave (cw) and time-resolved (TR) modes. The experiments have been performed employing specially designed samples containing the SL and an enlarged QW located far from the region where carriers are created by photopumping. A procedure of estimation of the basic kinetic parameters by fitting of the integrated cw and TR PL data is discussed.

\section{Experiment}

The structures are grown by molecular beam epitaxy pseudomorphically to a GaAs(001) substrate using the shutter-operation technique. All other growth conditions have been described elsewhere [4, 8]. A typical structure consists of a $20 \mathrm{~nm} \mathrm{ZnSe}$ buffer layer, a $0.6 \mu \mathrm{m}$ thick $\mathrm{Zn}_{0.93} \mathrm{Mg}_{0.07} \mathrm{~S}_{0.17} \mathrm{Se}_{0.83}$ bottom cladding layer, a 42 period $\left(4.2 \mathrm{~nm} \mathrm{ZnS}_{0.15} \mathrm{Se}_{0.85} / 1.2 \mathrm{~nm} \mathrm{Zn}_{0.87} \mathrm{Cd}_{0.13} \mathrm{Se}\right.$ ) SL with a $7 \mathrm{~nm} \mathrm{Zn}_{0.87} \mathrm{Cd}_{0.13} \mathrm{Se} \mathrm{QW}$ inserted in the middle, and, finally, a $0.12 \mu \mathrm{m}$ thick ZnMgSSe top cladding layer protected by a $5 \mathrm{~nm}$ ZnSe cap. The SL parameters were chosen to balance carefully alternating compressive and tensile strains between the $\mathrm{ZnCdSe}$ and $\mathrm{ZnSSe}$ constituent layers, in order to achieve a defect-free multilayer structure lattice-matched to the substrate as a whole.

PL measurements are performed in a helium flow cryostat permitting variation of the temperature in the $1.8-300 \mathrm{~K}$ range. The cw PL spectra are obtained with excitation by a $351 \mathrm{~nm}$ line of an Ar ion laser. PL excitation (PLE) characterization is performed using the emission of a tungsten lamp, dispersed by a single-grating monochromator. TR PL measurements are carried out using a frequency-doubled beam of an $\mathrm{Al}_{2} \mathrm{O}_{3}$ :Ti mode-locked laser pumped by a cw $\mathrm{Ar}^{+}$ laser, and a synchroscan streak camera. The pulse width of the beam is $0.4 \mathrm{ps}$ and the time resolution of the whole setup is estimated as about $15 \mathrm{ps}$. The excitation intensities are kept below a time-integrated power of $1 \mathrm{~W} / \mathrm{cm}^{2}$ by a neutral density filter.

\section{Results and discussions}

The low-temperature $(5 \mathrm{~K}) \mathrm{cw}$ PL spectrum shown in the inset of Fig. 1 contains two separated peaks. The PLE spectra enable one to attribute these lines to excitonic emission in the SL (the higher energy line) and in the wider QW. Figure 1 displays an integrated intensity of the PL lines from the SL (circles) and the QW (squares) as a function of temperature. The intensity of the PL line 


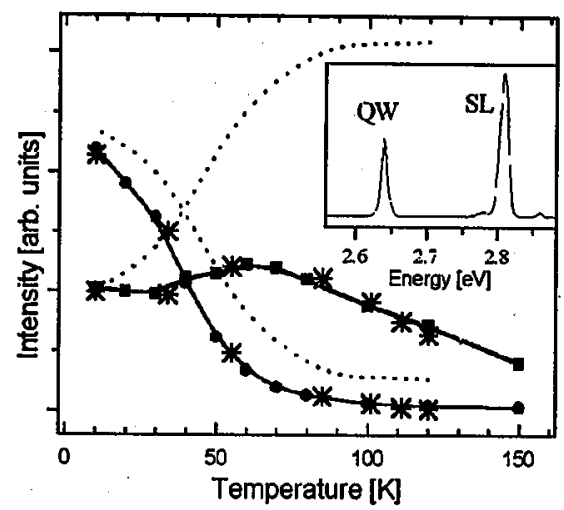

Fig. 1. Normalized integrated PL intensity from QW (squares) and SL (circles) at $5 \mathrm{~K}$. Solid lines are guided by the eye. The dotted curves present the fitting results at $\tau_{\mathrm{n}-\mathrm{rad}} \gg \tau_{\mathrm{rad}}$. The inset displays the $\mathrm{cw}$ PL spectrum at $5 \mathrm{~K}$.

associated with the SL decreases dramatically with an increase in temperature and disappears completely at about $100 \mathrm{~K}$. In contrast to that, the temperature dependence of the QW PL is non-monotonous with a peak at about $60-70 \mathrm{~K}$. Note that this line is well visible up to room temperature.

The TR PL spectra also demonstrate two well-distinguished bands attributed to SL and QW, respectively, and they also varied with temperature in a specific way. Integrated decay curves of the QW (solid line) and SL (dashed line) PL bands are presented in Fig. 2 for three temperatures. At low temperature the decay process in the SL is slower than in the QW. However, the temperature increase results not only in a redistribution between the QW and SL integrated PL intensities but in their decay times as well. The change of the SL decay curves indicates a faster decay process in SL at high temperature.

This PL behavior is most likely connected with a carrier transfer mechanism in the SL. To elucidate the driving forces we calculated the band line-ups and confinement energies in the SL samples using material parameters given in Ref. [9] and assuming pseudomorphic growth of the structure. The calculated miniband widths are $72 \mathrm{meV}, 9 \mathrm{meV}$, and $104 \mathrm{meV}$ for electrons, heavy holes, and light holes, respectively. At low temperatures the lowest heavy-hole exciton is essentially localized in the SL wells due to the low heavy-hole mobility along the SL axis, which explains the dominance of the SL PL line. However, the bottom of the light-hole miniband in this structure is only $5-10 \mathrm{meV}$ above the top of the heavy-hole one, providing the possibility for efficient thermal occupation of the light-hole states at higher temperature. This phenomenon may cause an enhanced transfer of holes along the growth direction, followed by their capture into the QW and energy relaxation down to the lowest heavy-hole QW level. As a result the temperature-induced increase in the QW PL intensity is expected. Note that at higher temperatures the QW PL intensity decreases again due to the enhanced contribution of non-radiative recombination channels. 


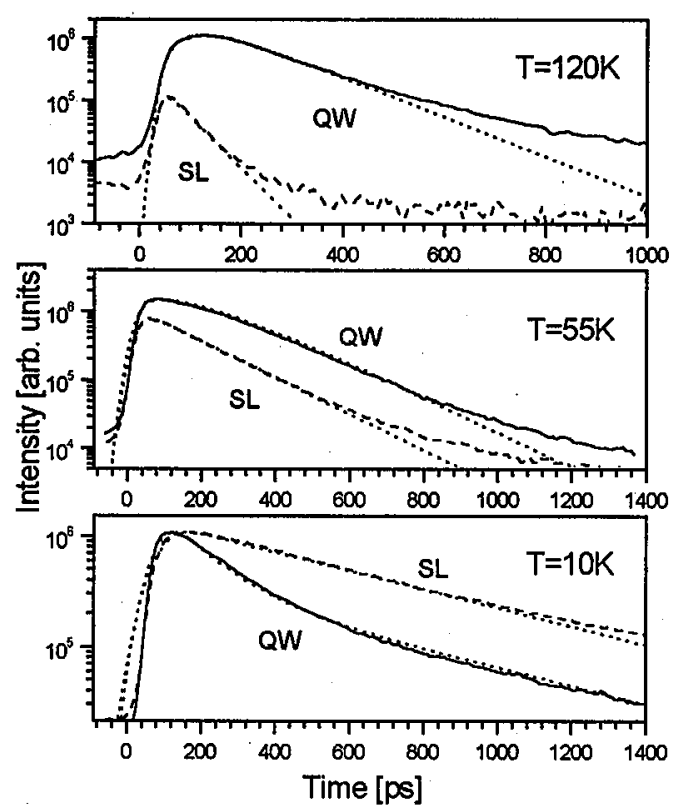

Fig. 2. Normalized integrated decay curves of QW (solid line) and SL (dashed line) PL bands measured at different temperatures. The dotted curves are the results of a fitting neglecting nonradiative processes.

To analyze the obtained data quantitatively we used a rate equation model [6] which describes the carrier kinetics in a two-level system as follows:

$$
\begin{aligned}
& \frac{\mathrm{d} N^{\mathrm{SL}}}{\mathrm{d} t}=G^{\mathrm{SL}}(t)-\frac{N^{\mathrm{SL}}}{\tau^{\mathrm{SL}}}, \quad \frac{1}{\tau^{\mathrm{SL}}}=\frac{1}{\tau_{\mathrm{rad}}^{\mathrm{SL}}}+\frac{1}{\tau_{\mathrm{n}-\mathrm{rad}}^{\mathrm{SL}}}+\frac{1}{\tau_{\mathrm{tr}}^{\mathrm{SL}-\mathrm{QW}}}, \\
& \frac{\mathrm{d} N^{\mathrm{QW}}}{\mathrm{d} t}=G^{\mathrm{QW}}(t)+\frac{N^{\mathrm{SL}}}{\tau_{\mathrm{tr}}^{\mathrm{SL}}-\mathrm{QW}}-\frac{N^{\mathrm{QW}}}{\tau^{\mathrm{QW}}}, \quad \frac{1}{\tau^{\mathrm{QW}}}=\frac{1}{\tau_{\mathrm{rad}}^{\mathrm{QW}}}+\frac{1}{\tau_{\mathrm{n}-\mathrm{rad}}^{\mathrm{QW}}},
\end{aligned}
$$

where $N^{\mathrm{SL}}$ and $N^{\mathrm{QW}}$ are the hole densities in the SL and QW, $G^{\mathrm{SL}}(t)$ and $G^{\mathrm{QW}}(t)$ are the generation of holes by exciting pulses in the SL and $\mathrm{QW}$, and $\tau^{\mathrm{SL}}$ and $\tau^{\mathrm{QW}}$ are the decay times in the SL and QW, respectively, $\tau_{\mathrm{rad}}$ and $\tau_{\mathrm{n}-\mathrm{rad}}$ are the radiative and nonradiative recombination lifetimes, correspondingly, $\tau_{\mathrm{tr}}^{\mathrm{SL}}-\mathrm{QW}$ is the transfer time between the SL and the QW.

The integrated intensities of the PL are written in this model as follows:

$$
\begin{aligned}
I_{\mathrm{SL}} \sim \frac{N^{\mathrm{SL}}}{\tau_{\mathrm{rad}}^{\mathrm{SL}}} & =G^{\mathrm{SL}} \frac{\tau_{\mathrm{n}-\mathrm{rad}}^{\mathrm{SL}}\left(\tau_{\mathrm{tr}}^{\mathrm{SL}-\mathrm{QW}}-\tau^{\mathrm{SL}}\right)-\tau^{\mathrm{SL}} \tau_{\mathrm{tr}}^{\mathrm{SL}-\mathrm{QW}}}{\tau_{\mathrm{n}-\mathrm{rad}}^{\mathrm{SL}} \tau_{\mathrm{tr}}^{\mathrm{SL}-\mathrm{QW}}} \approx G^{\mathrm{SL}}-G^{\mathrm{SL}} \frac{\tau^{\mathrm{SL}}}{\tau_{\mathrm{tr}}^{\mathrm{SL}-\mathrm{QW}}} \\
I_{\mathrm{QW}} & \sim \frac{N^{\mathrm{QW}}}{\tau_{\mathrm{rad}}^{\mathrm{QW}}}=\left(G^{\mathrm{QW}}+G^{\mathrm{SL}} \frac{\tau^{\mathrm{SL}}}{\tau_{\mathrm{tr}}^{\mathrm{SL}-\mathrm{QW}}}\right) \frac{\left(\tau_{\mathrm{n}-\mathrm{rad}}^{\mathrm{QW}}-\tau^{\mathrm{QW}}\right)}{\tau_{\mathrm{n}-\mathrm{rad}}^{\mathrm{QW}}} \\
& \approx G^{\mathrm{QW}}+G^{\mathrm{SL}} \frac{\tau^{\mathrm{SL}}}{\tau_{\mathrm{tr}}^{\mathrm{SL}-\mathrm{QW}}}
\end{aligned}
$$


The fitting procedure operates with both $\mathrm{cw}$ and TR data and includes some iterations starting from fitting without taking into account the nonradiative recombination to obtain approximate decay parameters. These parameters are used in the final fitting (including nonradiative process), permitting one to refine the decay values and obtain radiative and nonradiative recombination lifetimes.

The results of fitting the PL TR decay curves, with the assumption that $\tau_{\mathrm{n}-\mathrm{rad}} \gg \tau_{\mathrm{rad}}$ are presented in Fig. 2 by dotted lines. At $10 \mathrm{~K}$ the hole kinetics is characterized by decay times of about $520 \mathrm{ps}$ (SL) and $111 \mathrm{ps}$ (QW). At $120 \mathrm{~K}$ the values are $53 \mathrm{ps}$ and $133 \mathrm{ps}$, respectively. The tenfold shortening of the SL decay time together with the weakly-varied QW decay time confirms the existence of an effective transfer mechanism. The fitting of the $\mathrm{cw}$ integrated intensity neglecting the nonradiative processes is shown in Fig. 1 by dotted curves. Good agreement with experimental data is observed only at the lowest temperature. A better fit (marked by stars) is obtained using the refined parameters and taking account of the recombination processes.

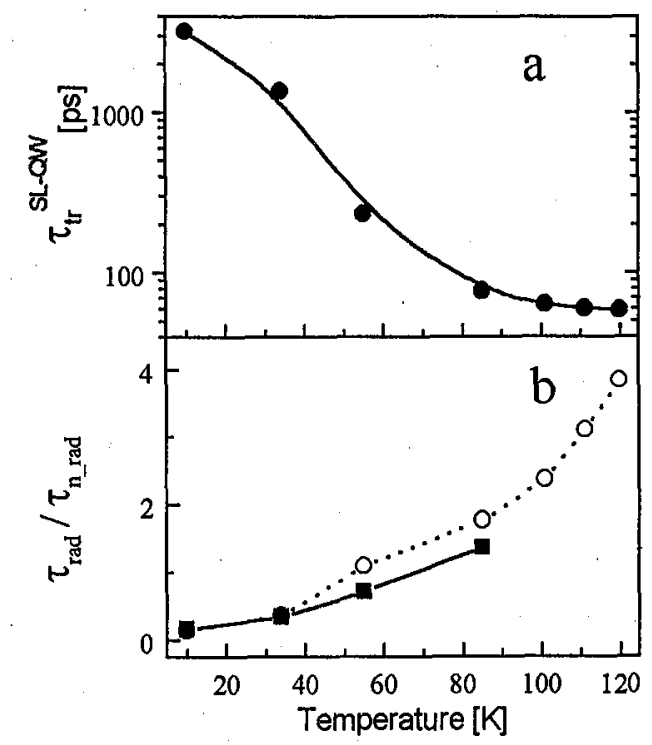

Fig. 3. Temperature dependences of the hole transfer time from SL to QW (a) and the ratio of the radiative recombination lifetime to the nonradiative one (b), for SL (solid squares) and $\mathrm{QW}$ (open circles).

As results of the complete fitting procedure we obtain the temperature dependence of the transfer time of holes from the SL to the QW (Fig. 3a). This value decreases drastically down to $c a .40 \mathrm{ps}$ at about $120 \mathrm{~K}$, indicating a temperature-induced transition from the slow "hopping" to an efficient Bloch-type transfer mechanism. Figure $3 \mathrm{~b}$ presents the temperature dependences of the radiative to nonradiative recombination lifetime ratio for SL (solid squares) and QW (open circle). Contrary to the monotonous increase in this value for SL the 
QW lifetime ratio reveals a fast rising and some hint of a plateau in the $50-80 \mathrm{~K}$ range. This agrees well with the peak appeared in the temperature dependence of the integrated QW PL (Fig. 1). The latter reflects the simultaneous enhancement of nonradiative recombination and hole transfer due to matching of lightand heavy-hole minibands in the SL.

In summary, we have experimentally observed an efficient vertical hole transport in the alternatively-strained $\mathrm{ZnCdSe} / \mathrm{ZnSSe}$ short-period SL. A quantitative description is worked out for the carrier kinetics in the SL structure with an enlarged QW (a typical system for the active region of optically pumped lasers). The simultaneous fitting of integrated cw PL spectra and decay curves has allowed us to obtain a complete set of parameters describing the process. The studied $\mathrm{ZnSSe} / \mathrm{ZnCdSe}$ SLs are found to be well applicable as highly-transparent hole emitters efficiently operating at $300 \mathrm{~K}$ due to the temperature-enhanced transport within the light-hole miniband.

\section{Acknowledgment}

This work has been supported in part by the Russian Foundation for Basic Research (97-02-18269a and 98-02-18206) and by the Program of Ministry of Science of RF "Physics of Solid-States Nanostructures" (projects 97-2014 and 97-1035).

\section{References}

[1] A.A. Toropov, S.V. Ivanov, T.V. Shubina, A.V. Lebedev, L.M. Sorokin, S.V. Sorokin, G.N. Aliev, M.G. Tkatchman, N.D. Il'inskaya, P.S. Kop'ev, in: Proc. Int. Symposium, Nanostructures: Physics and Technology, St. Petersburg 1997, Eds. Zh. Alferov, L. Esaki, A.F. Ioffe Physico-Technical Institute, St. Petersburg 1997, p. 210.

[2] T.V. Shubina, S.V. Ivanov, A.A. Toropov, G.N. Aliev, M.G. Tkatchman, S.V. Sorokin, N.D. Il'inskaya, P.S. Kop'ev, J. Cryst. Growth 184/185, 596 (1998).

[3] S. Sorokin, S. Ivanov, A. Toropov, T. Shubina, I. Sedova, M. Tkatchman, P. Kop'ev, Zh. Alferov, in Ref. [1], p. 206.

[4] S. Ivanov, A. Toropov, S. Sorokin, T. Shubina, N. Il'inskaya, A. Lebedev, P. Kop'ev, Zh. Alferov, H.-J. Lugauer, G. Reuscher, M. Keim, F. Fischer, A. Waag, G. Landwehr, Semiconductors, to be published.

[5] A. Chomette, B. Deveand, A. Regreny, G. Bastard, Phys. Rev. Lett. 57, 1464 (1986).

[6] A. Nakamura, K. Fujiwara, Y. Tokuda, T. Nakayama, M. Hirai, Phys. Rev. B 34, 9019 (1986).

[7] N. Kawamoto, A. Nakamura, K. Fujiwara, Jpn. J. Appl. Phys. 28, L1715 (1989).

[8] S.V. Ivanov, S.V. Sorokin, P.S. Kop'ev, J.R. Kim, H.D. Jung, H.S. Park, J. Cryst. Growth 159, 16 (1996).

[9] C.G. Van de Walle, Phys. Rev. B 39, 1871 (1989). 